\title{
EDITORIAL
}

\section{Which heart valve prosthesis for patients aged between 60 and 70 years?}

\section{G Hanania}

Heart 2003;89:481-482

The advantages and disadvantages of the two types of heart valves - mechanical and bioprostheses - can pose a dilemma when it comes to choosing the most appropriate treatment for patients aged between 60 and 70 years

Correspondence to: Dr Guy Hanania, Service de Cardiologie, Centre Hospitalier Robert Ballanger, 93602 Aulnay-sous-Bois Cedex, France;

cardio@ch-aulnay.fr
$\mathrm{P}$ osthetic valve replacement has transformed the prognosis of major and poorly tolerated valvulopathies. The respective advantages and disadvantages of the two types of valves available are well known: mechanical prostheses (MP) theoretically have an indeterminate life span but carry the risk of thromboembolic events that require anticoagulant therapy, which itself is responsible for haemorrhages; bioprostheses (BP), free of anticoagulant treatment and thus haemorrhagic risk, bear a theoretically lower thromboembolic risk (but in fact equal to that of an anticoagulated MP) and have a limited life span that requires reintervention at a subsequent date influenced by the patient's age at implantation, the valve replaced, and the BP type used.

These respective risks affect the long term outcome of the prosthesis bearer and differ by their time of occurrence. Thromboembolic and haemorrhagic events can occur at any time during the postoperative period, whereas BP deterioration, highly unusual during the first few years after implantation, can occur as early as the 10th year or even earlier, especially in patients younger at the time of surgery and those with mitral valvulopathy, and is seen in almost all patients by 20 years.

The indication for a BP must take into account the contraindications for anticoagulants, the patient's informed consent, and his/her life expectancy at the time of the intervention. This latter essential factor explains the preference for an MP before 60 years and a BP after 70 years. However, a consensus has not yet been reached for the seventh decade, between 60 and 70 years, even though the American College of Cardiology/ American Heart Association recommendations ${ }^{1}$ set the respective age limits for aortic and mitral bioprostheses at 65 and 70 years.

\section{LONG TERM OUTCOMES}

Good long term outcomes have been obtained with these different replacement valves, but comparisons between MP and BP are less common. The Edinburgh heart valve trial ${ }^{2}$ randomised 533 patients, 261 with mitral and 211 with aortic valvulopathies, to receive either a Bjork MP, or a Hancock or Carpentier-Edwards porcine BP. After
20 years of follow up, no difference between the survival rates of the two groups was observed. The reoperation rate was higher for the $\mathrm{BP}$ group, while more patients with MP haemorrhaged.

The Veterans Affairs (VA) trial ${ }^{3}$ randomised 575 men to receive either a Bjork or Hancock prosthesis with 394 aortic and 181 mitral replacements. With 15 years of follow up, survival was better for patients receiving an aortic MP than a BP and was comparable for mitral valve replacements. Haemorrhages were more common with mechanical valves, but no significant difference was noted for thromboembolic and infectious complications. Notably, BP deteriorated even more frequently when the subject was younger at the time of surgery: $26 \%$ before 65 years and $9 \%$ thereafter for aortic replacements, and $44 \%$ for mitral valves in patients under 65 years.

A retrospective French study ${ }^{4}$ on a highly homogeneous cohort, defined by their year of intervention (1985), length of follow up, and age at the time of surgery between 60 and 70 years (all born between 1915 and 1925), gave results similar to those reported by the VA. Among the 574 patients enrolled, 497 had monovalvar replacements: 313 aortic (160 MP and $153 \mathrm{BP}$ ) and 184 mitral (99 MP and $85 \mathrm{BP}$ ).

Overall survival was better for the patients receiving MP (46\%) than porcine valves $(32 \%)$ to replace defective aortic valves, and rates were equivalent in the mitral position $(34 \%$ and $33 \%$, respectively).

The BP deterioration rate at 15 years was $48 \%$ for mitral valves versus $20 \%$ for aortic valves, representing $28.2 \%$ and $8.5 \%$, respectively, of the initially operated population. The percentages of aortic and mitral BP deterioration varied as a function of the patient's age at the time of implantation: $13.5 \%$ and $32.6 \%$ before 65 years, $3.8 \%$ and $22.2 \%$ thereafter, respectively. Postoperative mortality for reoperated patients was $9 \%$.

The rates of thromboembolic events were comparable for mitral MP and BP (19\%) versus only $5 \%$ for aortic BP and $8 \%$ for MP. Finally, haemorrhages clearly occurred more frequently in MP bearers-15\% of aortic and $12 \%$ of mitral valves-as opposed to $6 \%$ for mitral $\mathrm{BP}$, more commonly associated with anticoagulant treatment, and $2 \%$ for aortic BP.

\section{ELDERLY PATIENTS}

BP tend to be preferred for elderly patients, ${ }^{5}$ but some authors have provided favourable long term

Abbreviations: $\mathrm{BP}$, bioprosthesis; INR, international normalised ratio; MP, mechanical prosthesis; VA, Veterans Affairs 
results for bileaflet valves. ${ }^{6}$ The weak thrombogenicity of the latter, the possibility of moderate anticoagulation ${ }^{78}$ for aortic prostheses with an international normalised ratio (INR) of $2-3$, the foreseeable widespread use of self testing to control anticoagulation, ${ }^{9}$ and the frequent indications of oral anticoagulant for atrial fibrillation associated with mitral valve replacements are all arguments supporting the extension of MP use to elderly subjects.

On the other hand, valid arguments favour retaining BP as the reference aortic prosthesis for aged individuals: no need for anticoagulant treatment, which is always difficult to pursue at an age when extracardiac disease(s) is ever more common, and low frequency of deterioration before the 15th year post-surgery. Henceforth, the choice will be guided by life expectancy at the time of the intervention and the surgical risk of reoperation for BP deterioration in the elderly subject. In France, life expectancy predictions for women and men, respectively, are 25.7 and 19.5 years at 60 years of age, 20.4 and 16 years at 65 , and 16.3 and 12.7 years at 70 .

Based on the reported deterioration rates of mitral BP, they do not appear to be good replacement valves in this position before 70 years of age. The seventh decade thus remains the domain of the MP for mitral valve replacement.

For aortic valvulopathies, the choice is more subtle. For valves replaced before 65 years, the reintervention rate around 80 years remains high, affecting close to $10 \%$ of patients. Furthermore, this percentage will continue to rise because life expectancy in developed countries, where it was expected to stabilise, has continued to increase. The elderly subject's risk of reoperation has been estimated to be $6-11 \% .{ }^{10}{ }^{11}$ Morbidity attributable to these reinterventions is much higher, affecting $34 \%$ of the reoperated population. ${ }^{11}$ After 65 years, the possible need for reintervention because of deterioration is lower and the aortic BP can be preferred over the MP for certain patients.

\section{SUMMARY}

The seventh decade is, in terms of valve replacement, a transitional period during which the choice between MP and BP must take into consideration multiple parameters that determine the long term postoperative outcome.

- The thromboembolic risk is theoretically equivalent for both valve substitutes (under oral anticoagulation for MP and without for BP); however, an added risk exists for the former should extracardiac surgery, frequent at these ages, be necessary.

- Anticoagulant treatment for MP and/or atrial fibrillation bears an inherent haemorrhagic risk and, when prolonged beyond 80 years, increases the frequency and severity of such events, even though the use of moderate anticoagulation is authorised for currently available aortic MP and the imminent development of self testing promises to lower this risk.

- The predictable time at which a primary BP will fail is even longer for the older subject and when the aortic position is involved.
- Life expectancy of the subject should be less than the foreseeable deterioration of the replacement valve.

- The risk of reintervention for prosthesis deterioration at 80 years and older must be taken into consideration.

- The patient's own characteristics-extracardiac disease(s) shortening life expectancy or contraindicating anticoagulants, impossibility of achieving optimal treatment monitoring and, on the other hand, indication of anticoagulant treatment for atrial fibrillation-must be entered into the equation.

- Finally, the patient has to make his or her own decision, after being fully informed of the two therapeutic options.

At the end of this analysis, and in light of the results reported in the literature, the logical choice for mitral valve replacement before 70 years is an MP. For an aortic valve and before 65 years, today's life expectancy is such that an MP remains the best solution to avoid reintervention, which is always more risk laden than primary surgery in an octogenarian. Between 65 and 70 years, the choice is less restricted. The preference should continue, especially in women endowed with a longer mean life span, to favour MP with the possibility of moderate anticoagulant treatment (INR of 2-3.5) without excessive morbidity over a prolonged postoperative period, including essentially the eighth decade.

\section{REFERENCES}

1 Bonow RO, Carabello B, de Leon AC Jr, et al. ACC/AHA guidelines for the management of patients with valvular heart disease. J Am Coll Cardiol 1998; 32: 1486-588.

2 Bloomfield P, Wheatley DJ, Prescott RJ, et al. Twelve-year comparison of a Biork-Shiley mechanical heart valve with porcine bioprostheses. N Engl J Med 1991;324:573-9.

3 Hammermeister K, Sethi GK, Henderson WG et al. Outcomes 15 years after valve replacement with a mechanical versus a bioprosthetic valve: final report of the Veterans Affairs randomized trial. J Am Coll Cardiol 2000;36: 1 152-8.

4 Hanania G, Michel PL, Montely JM, et al. Outcomes 15 years after valve replacement with a mechanical versus a bioprosthetic valve in patients between 60 and 70 years of age. J Am Coll Cardiol 2002;39(suppl A):423A

5 Holper K, Wottke M, Lewe T, et al. Bioprosthetic and mechanical valves in the elderly: benefits and risks. Ann Thorac Surg 1995;60(suppl. 2):S443-6.

6 Emery RW, Arom KV, Kshettry VR. Decision-making in the choice of heart valve for replacement in patients aged 60-70 years: twenty-year follow up of the St. Jude Medical Aortic Valve Prosthesis. J Heart Valve Dis 2002;11/suppl I):S37-44.

7 Gohlke-Barwolf C, Acar J, Oakley C, et al. Guidelines for prevention of thromboembolic events in valvular heart diseases: study group of the working group on valvular heart disease of the European Society of Cardiology. Eur Heart J 1995;16:1320-30.

8 Acar J, lung B, Boissel JP, et al. AREVA: multicenter randomized comparison of low-dose versus standard-dose anticoagulation in patients with mechanical prosthetic heart valves. Circulation 1996;94:2107-12.

9 Rosengart TK. Anticoagulation self testing after heart valve replacement. J Heart Valve Dis 2002;11 (suppl. I):S61-5.

10 Khan S. Long-term outcomes with mechanical and tissue valves. J Heart Valve Dis 2002;11 (suppl 1):S8-14.

11 Dalrymple-Hay MJR, Crook T, Bannon PG, et al. Risk of reoperation for structural failure of aortic and mitral tissue valves. J Heart Valve Dis 2002;11:419-23. 